\title{
A Comparative Analysis of Foundations using Prescriptive Design and Static Loading Test Methods
}

\author{
(Case Study: The Karuma Interconnection Power Project in Uganda) \\ *Acidri Samuel ${ }^{1}$, Kyakula Michael ${ }^{2}$, and Mugume Rodgers Bangi ${ }^{3}$ \\ ${ }^{1,2,3}$ Faculty of Engineering, Department of Civil and Building Engineering, Kyambogo University, Uganda \\ *Corresponding Author,
}

\begin{abstract}
Despite recent improvements in soil characterisation, geotechnical exploration and construction methodologies, 66.7\% of overhead transmission line foundation design engineers use prescriptive design methods with applied traditional factors of safety between 2.5 to over 4.0, to design foundations in the face of uncertainties in ground conditions and design criteria, non-linear nature of the load-displacement response of foundations and the prescriptive design's tendency to give linear solutions. Hence, the use of full-scale foundations and static load tests to assess the overall response of foundations. A $6900 \mathrm{~mm}$ pile and 3 pad foundations along Uganda's $400 \mathrm{kV}$ Karuma Interconnection Project were designed, constructed and tested under uplift, compression and lateral loads as per the respective failure modes. The results suggested that the maximum displacements were within $0.36-18.96 \%$ of the prescriptive $25 \mathrm{~mm}$ value for uplift, $3.32 \%$ of the prescriptive 25 mm value for compression, and $4.78 \%$ of the prescriptive $50 \mathrm{~mm}$ value for the lateral load test in conformity to IEC 61773 (1996) and COMESA/FDHS 293 (2007).

The foundations' insitu load capacities from the hyperbolic graphs as per the Chin-Kondner extrapolation (1971), confirmed that the foundations could adequately resist the working loads at $100 \%$ and ultimate design loads at $130 \%$, despite uncertainties of moderately aggressive chemical environment exposures as per BS EN 206 (2013) or soils with medium to high degrees of plasticity with low swell potentials.
\end{abstract}

Keywords: Foundations, Prescriptive Design, Static Load Tests (SLTs), Transmission Towers

\section{INTRODUCTION}

\subsection{The Local Geology}

The geology of Karuma Interconnection Project mainly consists of quaternary rock systems of laterite, alluvium, swamp and lacustrine deposits, Neoproterozoic rock systems of mudstones, shales, slates and phyllites, Mesoproterozoic rock system of mica schists, and Neoarchaean rock systems of metagabbro, porphyritic-granite, meta-dolerite, granodiorite, biotite-hornblende and banded gneiss.

\subsection{The Research Background}

Foundation substructures are essential structural members that transmit and distribute different kinds of superstructure loads to the substrata below without exceeding the bearing capacity of the ground and preventing excessive or uneven settlements, and they are generally classified as shallow or spread and deep foundations [1-3]. Foundations must fulfil both structural and geotechnical parameters, but due to uncertainties of the subsoil behaviour, most foundations are either statically or dynamically tested to verify conformity with the design load, but the latter is not commonly used in Southern Africa [4,5].

In Uganda, since 2013, load tests have been used to either prove the maximum capacity of the foundation and/or to verify the predicted design values and settlements under pressure on a foundation that is expendable to the main works; and at times on a working pile whilst limiting the maximum test load to less than 1.5 times the safe working load [5-7].

Foundation design entails that neither the foundation units collapse nor should they induce the overall shear failure of the supporting ground, lest the foundation's post-construction settlement values exceed the permissible tolerances in the codes and specifications [8-11]. The design of foundations consists of proportioning the foundation, mitigating limit state conditions such as the ultimate limit state properties of loss of static equilibrium of the structure, failure by collapse or by fatigue; and/or the serviceability limit state properties of deflection, cracking, vibration, and deterioration of the foundation structure [3,12-14].

Uncertainties in geotechnical models and parameters and their effect have long been recognised [15-23]; and hence, to perform geotechnical and foundation designs using the prescriptive design approaches, conservative values of the uncertain soil parameters are often adopted along with an 'experience-calibrated' factor of safety $[11,24]$. In the quest to obtain a more rational design, many researchers such as Wu [25], Christian [26], Whitman [18], Phoon [27,28], Fenton [29], Najjar and Gilbert [30], Wang [31], and Zhang [32] have turned to reliability-based designs such as foundation full-scale models, analyses and foundation tests, which creates a much more-realistic geotechnical and foundation design outcome and is better in quantifying the uncertainties in soil parameters than the prescriptive design approaches [24]. Therefore, since also soils can soften or harden upon shearing and have a much more complex response than perfect plasticity; the recent focus on using the realistic full-scale foundation models, provides important insights into the overall response of foundations [33-38]. 


\section{GEOTECHNICAL INVESTIGATIONS}

\subsection{The Test Trial Pits and Borehole pits}

Four test pits were carefully located at a $1 \mathrm{~m}$ distance from the survey mark stones, and manually excavated to $1 \mathrm{~m} \times 0.5 \mathrm{~m}$ $\mathrm{x} 3 \mathrm{~m}$ dimensions for investigating the stratification of subsurface layers and ground water as per BS 5930: 1999+A2: 2010 and BS 6031: 2009. Obtained soil samples were labelled and placed in air-tight plastic bags for indicative laboratory soil tests.

\subsection{The Dynamic Penetration Light Test}

The DPL test was used in the determination of the soil's insitu resistance to the dynamic penetration of a cone, and in determining the soil's bearing capacity $(q)$ and resistance values using the Dutch formula below. Thus, the $N_{10}$ values were interpreted to give the respective granular and finegrained soil consistencies as per BS EN ISO 22476-2: 2005 + A1: 2011 and DIN 4094: 1990.

$\mathrm{q}=q_{d}=r_{d}\left[\frac{M}{(M+P)}\right]=\left[\frac{\mathrm{E}}{\mathrm{AxH}}\right] \times\left[\frac{\mathrm{M}}{(\mathrm{M}+\mathrm{P})}\right]$

$\mathrm{r}_{\mathrm{d}}=\frac{\mathrm{Mgh}}{\mathrm{Ae}}=\left[\frac{M g h \times N_{10}}{0.1 A}\right]=\frac{E}{A \times H}$

Where:

$q=$ soil's bearing capacity/dynamic point resistance

$r_{d}=$ soil's unit point resistance; and $E=\mathrm{Mgh}$

$A=$ cone area $\left(\mathrm{A}=0.001 \mathrm{~m}^{2}\right) ; H=$ penetration depth

$M=$ mass of hammer $(\mathrm{M}=10.252 \mathrm{~kg})$

$P=$ total assembly mass where the DPL assembly mass is $6.714 \mathrm{~kg}$, and each rod is $2.86 \mathrm{~kg}$

$N_{10}=$ blows per $10 \mathrm{~cm}$ penetration

$e=$ penetration rate $=0.1 / N_{10}$

\subsection{The Standard Penetration Test}

The SPT test was conducted in accordance with BS EN ISO 22476-3:2005 and ASTM D1586-99: 1999 to compute allowable bearing capacities from the corrected SPT $\mathrm{N}^{\prime}{ }_{55}$ values using the Terzaghi's formula (1967) and Bowles's (1982) approach based on Meyerhof's (1963) equations.

The Terzaghi's (1967) formulae is given below:

$\mathrm{N}^{\prime}{ }_{55}=\mathrm{C}_{\mathrm{N}} \times \mathrm{N} \times \eta_{1} \times \eta_{2} \times \eta_{3} \times \eta_{4}$

$\mathrm{C}_{\mathrm{N}}=\left(\frac{\mathrm{p}^{\prime \prime}{ }_{\mathrm{o}}}{\mathrm{p}_{\mathrm{o}}{ }_{\mathrm{o}}}\right)^{\frac{1}{2}}$ for $0.4 \leq \mathrm{C}_{\mathrm{N}} \leq 1.7$

$\mathrm{q}_{\mathrm{ult}}=5.14 \times \frac{\mathrm{qu}_{\mathrm{u}}}{2}=\left[\frac{5.14 \times 13.1 \times \mathrm{N}^{\prime}{ }_{55}}{2}\right]$

$\mathrm{q}_{\mathrm{all}}=\frac{\mathrm{q}_{\mathrm{ult}}}{\mathrm{FS}}=\frac{5.14 \times \mathrm{xc}_{\mathrm{u}}}{\mathrm{FS}}=\left\{\frac{5.14 \times 13.1 \times \mathrm{NN}^{\prime}{ }_{55}}{2 \times \mathrm{FS}}\right\}$

Where:

$\mathrm{q}_{\text {all }}=$ allowable bearing capacity

$\mathrm{C}_{\mathrm{N}}=$ adjustments for overburden pressure

$\mathrm{p}^{\prime}{ }_{\mathrm{o}}=$ overburden pressure

$\mathrm{p}^{\prime \prime}{ }_{\mathrm{o}}=$ reference overburden pressure $(95.76 \mathrm{kPa})$

$\mathrm{N}=$ corrected SPT $\mathrm{N}^{\prime}{ }_{55}$ values

$\eta_{1}=E_{r} / E_{r b}$ (hammer efficiency correction)

$\mathrm{E}_{\mathrm{r}}=$ average energy ratio

$\mathrm{E}_{\mathrm{rb}}=$ standard energy ratio

$\eta_{2}=$ rod length correction $\eta_{3}=$ sampler correction

$\eta_{4}=$ borehole diameter correction

FOS $=$ factor of safety

Bowles's (1982) approach based on Meyerhof's (1963) equations is given below:

$\mathrm{q}_{\text {all }}=\left\{\frac{\mathrm{N}}{\mathrm{F}_{2}}\left[\frac{\left(\mathrm{B}+\mathrm{F}_{3}\right)}{\mathrm{B}}\right]^{2} x \mathrm{~K}_{\mathrm{d}}\right\}$ for $\mathrm{B}>\mathrm{F}_{4}$

$\mathrm{q}_{\mathrm{all}}=\left[\frac{\mathrm{N}}{\mathrm{F}_{1}} x \mathrm{~K}_{\mathrm{d}}\right]$ for $\mathrm{B} \leq \mathrm{F}_{4}$

$\mathrm{K}_{\mathrm{d}}=\left[1+\frac{0.33 \mathrm{D}}{\mathrm{B}}\right] \leq 1.33$

Where:

$\mathrm{q}_{\text {all }}=$ allowable bearing capacity

$\mathrm{N}=$ corrected $\mathrm{SPT} \mathrm{N}^{\prime}{ }_{55}$ values

$\mathrm{N}^{\prime}{ }_{55}=$ adjusted $\mathrm{N}$-values

$\mathrm{B}=$ foundation width/breadth

$\mathrm{F}_{1}=0.05 ; \mathrm{F}_{2}=0.08 ; \mathrm{F}_{3}=0.3 ;$ and $\mathrm{F}_{4}=1.2$

$\mathrm{D}=$ foundation depth

Meyerhof's (1963) equations are given below:

$\mathrm{q}_{\mathrm{a}}=0.73 \mathrm{~N}^{\prime \prime} \mathrm{R}_{\mathrm{D}_{1}} \times \mathrm{S}_{\mathrm{a}}(\mathrm{B} \leq 1.2 \mathrm{~m})$

$\mathrm{q}_{\mathrm{a}}=0.48 \mathrm{~N}^{\prime \prime} \mathrm{R}_{\mathrm{D}_{2}}\left(\frac{\mathrm{B}+0.3}{\mathrm{~B}}\right)^{2} \mathrm{~S}_{\mathrm{a}}(\mathrm{B}>1.2 \mathrm{~m})$

$\mathrm{R}_{\mathrm{D}_{1}}=1+0.2\left(\frac{\mathrm{D}_{\mathrm{f}}}{\mathrm{B}}\right) \leq 1.2$ for $\emptyset=0$

$\mathrm{R}_{\mathrm{D}_{2}}=1+0.1\left(\frac{\mathrm{D}_{\mathrm{f}}}{\mathrm{B}}\right) \leq 1.2$ for $\emptyset=0$

Where:

$\mathrm{q}_{\mathrm{a}}=\mathrm{q}_{\mathrm{all}}=$ allowable bearing capacity

$\mathrm{N}^{\prime \prime}=$ corrected SPT $\mathrm{N}^{\prime}{ }_{55}$ values

$\mathrm{R}_{\mathrm{D}_{1}}$ and $\mathrm{R}_{\mathrm{D}_{2}}=$ Meyerhof's depth reduction factors

$\emptyset=$ Internal angle of soil friction in degrees

$\mathrm{B}=$ foundation width/breadth (in metres)

$\mathrm{D}_{\mathrm{f}}=$ foundation depth

$S_{a}=$ Allowable settlement limit of $25 \mathrm{~mm}$

\subsection{Indicator Laboratory Soil Tests}

The obtained disturbed and undisturbed soil samples were tested for moisture content [39], particle size distribution [40,41], liquid limit [42], plastic limit and plasticity index [43], linear shrinkage limit [43], pH [44,45], sulphate and chloride content $[44,46]$, bulk density and unit weight $[43,47]$, specific gravity [43,48], direct shear [49] and onedimensional consolidation [50,51].

\subsection{Static Load Foundation Tests}

Static Load Tests are the most reliable and fundamental forms of in-situ loading tests, considered as the bench-mark of foundation performance and used for validating load capacities and design assumptions of the foundation regarding the axial compression or tension resistance, or its deflected shape under a lateral load. They involve the measurement of foundation head displacements in response to a physically applied test load until its failure point to replicate the long-term sustained load conditions [5,6,52]. They are standardised by ASTM D1143 for static axial compressive test; ASTM D3689 for static axial tensile (or uplift) test, and ASTM D3966 for lateral load test [6,53-56]. 


\subsubsection{The Static Axial Tensile Load Test}

As standardised by ASTM D3689/D3689M-07 (2013) and IEC 61773 (1996), the Static Axial Tensile load test was used for verifying the behaviour of vertical or batter tension foundations like those of overhead transmission lines with respect to their tensile capacity and axial stiffness, so as to provide the most reliable relationship between the static tensile load applied axially to a foundation and the resulting axial movements. Hence, the obtained information was used in assessing the foundation shaft's side shear resistance distribution, amount of end-bearing developed and the longterm load-deflection behaviour. It was also used to determine if the foundation had an ultimate static capacity and a deflection at service load satisfactory to support the specified foundation or superstructure $[53,57]$.

\subsubsection{The Static Axial Compressive Load Test}

The Static Axial Compressive load test measured the axial deflections of vertical or inclined foundations when loaded in static axial compression in order to confirm the foundation's structural and geotechnical reliability and to predict its settlement rate. The load is thus, increased in stages until the proposed working load and a certain factor of safety is reached, and then unloading the load until the rise or rebound has substantially ceased. The foundation may be tested in three cycles, whereby the first cycle is to $150 \%$ of foundation's Design Load (DL), the second cycle test is to $200 \%$ of DL and the third cycle tests the foundation to its ultimate load, defined as $250 \%$ to $300 \%$ of its DL [53,54]. Since the procedure leading up to $300 \%$ of the Design Load is very time consuming, the commonest method used stops at the first cycle and is limited to between $100 \%$ to $130 \%$ of the design load $[5,6,57]$.

\subsubsection{The Lateral Load Test}

As per ASTM D3966/D3966M-07 (2013), the Lateral load test was conducted to measure the lateral deflection of a vertical or inclined foundation when subjected to lateral loading, with the results used in characterising the variation of pile-soil interaction properties such as the coefficient of horizontal subgrade reaction, and estimation of bending stresses and lateral deflection over the pile's length for use in its structural design $[53,56]$.

\section{RESULTS AND DISCUSSIONS}

\subsection{The Test Trial Pits and Borehole pits}

Ground water tables were encountered above the base of footings in the swampy locations of KL 30 and AP 104/5 at $0.3 \mathrm{~m}$ and $1.14 \mathrm{~m}$ levels respectively; and ground water tables were encountered below the base of footings in locations AP $108 / 15$ and AP $108 / 20$ at levels of below $10 \mathrm{~m}$ and $4 \mathrm{~m}$ respectively below existing ground level [58]. The ground water table results in Table 1 , were used in computing the soil's effective unit weight $\left(\gamma^{\prime}\right)$, unit surcharge magnitude $(q)$ and corrections for water table effects on bearing capacities. Also, the insitu soil profile descriptions were used as a precursor assessment to the final soil grading and classifications as shown in Table 2 below.

$$
\begin{aligned}
& q=\gamma\left(D_{f}-D\right)+\gamma^{\prime} D \\
& q=\gamma \mathrm{D}_{\mathrm{f}} \\
& q=\gamma_{a v} \mathrm{D}_{\mathrm{f}}
\end{aligned}
$$

Where:

$\gamma=$ unit weight; and $\gamma^{\prime}=$ effective unit weight

$\gamma_{a v}=\frac{1}{B}\left[\gamma D+\gamma^{\prime}(B-D)\right]$ for $(D \leq B)$

$\gamma_{a v}=\gamma$ for $(D>B)$

Table 1: The insitu ground water table levels

\begin{tabular}{ccccc}
\hline Site & Depth $(\mathrm{m})$ & Pits & WT $(\mathrm{m})$ & FFL $(\mathrm{m})$ \\
\hline $1(\mathrm{PS})$ & 10.0 & BHP & 10.0 & 2.75 \\
\hline $2(\mathrm{GS})$ & 3.0 & TP & 4.0 & 3.50 \\
\hline $3(\mathrm{WL})$ & 10.0 & BHP & 1.14 & 4.50 \\
\hline $4(\mathrm{PL})$ & 20.0 & BHP & 0.30 & 12.8 \\
\hline
\end{tabular}

Note:

$1=$ AP 108/15; 2 = AP 108/20; 3 = KL 30; 4 = AP 104/5;

$\mathrm{PS}=$ Poor Soil $; \mathrm{GS}=$ Good Soil $; \mathrm{WL}=$ Waterlogged Location;

$\mathrm{PL}=$ Pile Location; $\mathrm{WT}=$ Water Table; FFL $=$ Foundation's

\begin{tabular}{|c|c|c|c|}
\hline Site & Depth (m) & Insitu soil strata & $\begin{array}{c}\text { Soil } \\
\text { Classification }\end{array}$ \\
\hline 1 (PS) & $2.45-4.2$ & $\begin{array}{l}\text { Grey-dense } \\
\text { clayey sand }\end{array}$ & $\begin{array}{l}\text { Silty sand } \\
\text { (SM) }\end{array}$ \\
\hline 2 (GS) & $0.1-3.5$ & $\begin{array}{l}\text { Brownish-orange } \\
\text { laterite* with } \\
\text { duricrust }\end{array}$ & $\begin{array}{l}\text { Clayey sand } \\
\text { with gravel } \\
\text { (SC) }\end{array}$ \\
\hline $3(\mathrm{WL})$ & $4.5-6.5$ & $\begin{array}{l}\text { Moist reddish brown, } \\
\text { mottled grey, hard } \\
\text { gravelly-clays }\end{array}$ & $\begin{array}{l}\text { Gravelly clays } \\
\text { of intermediate } \\
\text { plasticity (CI) }\end{array}$ \\
\hline $4(\mathrm{PL})$ & $12.7-15$ & $\begin{array}{l}\text { Slightly moist greyish } \\
\text { brown, medium- } \\
\text { dense clayey sand }\end{array}$ & $\begin{array}{l}\text { Clayey sand } \\
\text { (SC) }\end{array}$ \\
\hline \multicolumn{4}{|c|}{$\begin{array}{l}\text { Note: } \\
1=\text { AP } 108 / 15 ; 2=\text { AP } 108 / 20 ; 3=\text { KL } 30 ; 4=\text { AP } 104 / 5 ; \\
\text { PS = Poor Soil; GS = Good Soil; WL = Waterlogged Location; } \\
\text { PL = Pile Location } \\
\text { - "Medium-dense gravelly-sand } \\
\text { - SC and SM = Using the USCS soil classification system } \\
\text { - CI = Using the BS } 5930 \text { classification system }\end{array}$} \\
\hline
\end{tabular}

Formation Level; BHP = Borehole Pit; TP = Trial Pit

Table 2: The insitu soil strata descriptions

\subsection{The Dynamic Penetration Light (DPL)}

The DPL's $N_{10}$ readings of 10 to 54 under the respective penetration rates $(e)$, corresponded to granular soils of medium-dense consistency mainly coarse-grained sandy soils as shown in Tables 3 and 4 below $[59,60]$. DPL tests were done to determine the blows per $10 \mathrm{~cm}$ penetrations $\left(N_{10}\right)$, consistency descriptions, computations of unit point $\left(r_{d}\right)$ resistance, and dynamic point $\left(q_{d}\right)$ resistance/soil bearing capacity as per Eq. (1) and (2).

Table 3: The DPL result summary for AP 108/20

\begin{tabular}{cccccc}
\hline $\begin{array}{c}\text { Depth } \\
(\mathrm{m})\end{array}$ & $\begin{array}{c}\mathbf{M}_{\mathbf{1}} \\
(\mathrm{kg})\end{array}$ & $\boldsymbol{N}_{\mathbf{1 0}}$ & $\begin{array}{c}\mathrm{e} \\
(\mathrm{m} / \mathrm{blow})\end{array}$ & $\begin{array}{c}\boldsymbol{r}_{\boldsymbol{d}} \\
(\mathrm{MPa})\end{array}$ & $\begin{array}{c}\boldsymbol{q}_{\boldsymbol{d}} \\
(\mathrm{MPa})\end{array}$ \\
\hline 1.0 & 10.252 & 10 & 0.010 & 4.90 & 2.7 \\
\hline 2.0 & 10.252 & 54 & 0.002 & 26.46 & 13.2 \\
\hline 3.0 & 10.252 & 13 & 0.008 & 6.37 & 2.9 \\
\hline 3.5 & 10.252 & 14 & 0.007 & 6.86 & 2.9 \\
\hline
\end{tabular}




\section{Note:}

$\boldsymbol{M}_{\mathbf{1}}=$ mass of hammer; $\boldsymbol{N}_{\mathbf{1 0}}=$ blows per $10 \mathrm{~cm}$ penetration $\mathrm{e}=$ penetration rate ( $\mathrm{m}$ per blow); $\boldsymbol{r}_{\boldsymbol{d}}=$ unit point resistance $\boldsymbol{q}_{\boldsymbol{d}}=$ dynamic point resistance/soil bearing capacity

Table 4: Granular-soil consistency from DPL test

\begin{tabular}{cccc}
\hline $\begin{array}{c}\text { Blows, } \\
\mathrm{N}_{10}\end{array}$ & $\begin{array}{c}\text { Consistency } \\
\text { description }\end{array}$ & $\begin{array}{c}\text { Blows, } \\
\mathrm{N}_{10}\end{array}$ & $\begin{array}{c}\text { Consistency } \\
\text { description }\end{array}$ \\
\hline Less than 1 & Very Loose & $7-83$ & Medium Dense \\
\hline $1-7$ & Loose & Over 83 & Dense \\
\hline
\end{tabular}

Source: Nilsson (2012)

\subsection{The Standard Penetration Testing}

The SPT N-value of fine-grained soils at KL 30 of 100, corresponded to a hard soil consistency, whereas the SPT Nvalues of coarse-grained soils at locations AP 108/15 and AP $104 / 5$ were 24 and 27 respectively, corresponding to medium-dense soil consistencies as shown in Tables 5 to 7. The SPT results were used in determining the soil's preliminary consistency descriptions using the $\mathrm{N}$-values and parameters for bearing capacity analysis $[58,61]$ as shown in Eq. (3) to (13).

Table 5: The insitu SPT result summaries

\begin{tabular}{lcccc}
\hline Site & $\begin{array}{c}\text { SPT } \\
\text { N-value }\end{array}$ & $\begin{array}{c}\text { Corrected } \\
\mathrm{N}_{55}\end{array}$ & $\begin{array}{c}\text { Soil } \\
\text { consistency }\end{array}$ & $\begin{array}{c}\text { Soil } \\
\text { Classification }\end{array}$ \\
\hline 1 (PS) & 24 & 19 & $\begin{array}{c}\text { Medium- } \\
\text { dense }\end{array}$ & $\begin{array}{c}\text { Silty Sand } \\
\text { (SM) }\end{array}$ \\
\hline $3(\mathrm{WL})$ & 100 & 77 & Hard & $\begin{array}{c}\text { Gravelly clays } \\
\text { of intermediate } \\
\text { plasticity (CI) }\end{array}$ \\
\hline $4(\mathrm{PL})$ & 27 & 20 & $\begin{array}{c}\text { Medium- } \\
\text { dense }\end{array}$ & $\begin{array}{c}\text { Clayey Sand } \\
\text { (SC) }\end{array}$ \\
\hline Note: & & & &
\end{tabular}

$1=\mathrm{AP} 108 / 15 ; 2=\mathrm{AP} 108 / 20 ; 3=\mathrm{KL} 30 ; 4=\mathrm{AP} 104 / 5$;

PS = Poor Soil; GS = Good Soil; WL = Waterlogged; $\mathrm{PL}=$ Pile

- $\mathrm{SC}$ and SM = USCS soil classification system

- $\mathrm{CI}$ = BS 5930 soil classification system

Table 6: Consistency table for coarse-grained soils

\begin{tabular}{ccc}
\hline S/No & Consistency Description & SPT N-values \\
\hline 1 & Very Loose & Less than 4 \\
2 & Loose & 4 to 10 \\
3 & Medium-Dense / Compact & 10 to 30 \\
4 & Dense & 30 to 50 \\
5 & Very Dense & Over 50 \\
\hline
\end{tabular}

Source: BS 5930: $1999+$ A2: 2010

Table 7: Consistency table for fine-grained soils

\begin{tabular}{ccc}
\hline \multirow{2}{*}{ Description } & $\begin{array}{c}\text { Unconfined } \\
\text { Compressive Strength }(\mathrm{kPa})\end{array}$ & $\begin{array}{c}\text { SPT } \\
\text { N-value }\end{array}$ \\
\hline Very soft & Less than 25 & Less than 2 \\
Soft & 25 to 50 & 2 to 5 \\
Firm & 50 to 100 & 5 to 10 \\
Stiff & 100 to 200 & 10 to 20 \\
Very stiff & 200 to 380 & 20 to 40 \\
Hard & Over 380 & Over 40 \\
\hline
\end{tabular}

Source: BS 5930: $1999+$ A2: 2010

\subsection{The Soil Resistivity Testing}

The resistivity values showed that the soils at locations KL 30, AP 108/20 and AP 104/5 were essentially noncorrosive, whereas the soil at AP 108/15 was highly corrosive as shown in Tables 8 and 9 below. The soil resistivity test was used as a preliminary and non-conclusive test to provide generalised insitu environmental exposure conditions which may lead to steel depassivation and corrosion, and affect the structural design of the reinforced concrete foundations. Thus, for a more conclusive study, chemical tests in section 3.11, were deemed necessary regardless of the level of corrosiveness encountered [61,62].

Table 8: The insitu soil resistivity test results

\begin{tabular}{lcc}
\hline Site & $\begin{array}{c}\text { Average soil } \\
\text { resistivity }(\boldsymbol{\Omega m})\end{array}$ & $\begin{array}{c}\text { Soil corrosiveness description } \\
\text { (See Table 9 below) }\end{array}$ \\
\hline $1(\mathrm{PS})$ & 29.845 & Highly corrosive \\
\hline $2(\mathrm{GS})$ & 1201.472 & Essentially non-corrosive \\
\hline $3(\mathrm{WL})$ & 220.5 & Essentially non-corrosive \\
\hline $4(\mathrm{PL})$ & 285.192 & Essentially non-corrosive \\
\hline
\end{tabular}

Table 9: Resistivity explanations

\begin{tabular}{ccc}
\hline S/No & Soil Resistivity $(\Omega \mathrm{m})$ & Soil Corrosiveness \\
\hline 1 & Greater than 200 & Essentially non-corrosive \\
2 & $100-200$ & Mildly corrosive \\
3 & $50-100$ & Moderately corrosive \\
4 & $30-50$ & Corrosive \\
5 & $10-30$ & Highly corrosive \\
6 & Less than 10 & Extremely corrosive \\
\hline
\end{tabular}

Source: Roberge (2000)

\subsection{The Vane Shear Testing}

No vane shear tests (VST) were conducted in the borehole pits as the clay soils encountered were of a 'stiff to very stiff consistency' and not fitting the criteria for tests as per the requirements of ASTM D2573/D2573M-18 (2018). The VST method is not applicable for unsaturated or non-plastic silts, sands, gravels or other high permeability soils which dilate, collapse and generate pore pressures $[58,61,63,64]$.

\subsection{The Specific Gravity Testing}

The specific gravity $\left(G_{s}\right)$ values showed that the soils at location AP 108/15 were sand with silty particles, AP 108/20 had gravelly soil with clay mineral compositions, KL 30 had clay with gravel particles, and AP 104/5 had sand with clay compositions as shown in Tables 10 and 11. These descriptions are fairly comparable but inconclusive to the final descriptions by the USCS and BS 5930 soil classification systems as discussed in section 3.9. Thus, higher $G_{S}$ values give higher load bearing capacities since an increase in $G_{s}$ increases the soil's shear strength parameters and its suitability as a construction material [65-67]. 
Table 10: Insitu specific gravity summaries

\begin{tabular}{|c|c|c|c|}
\hline Site & $\begin{array}{c}\text { Gs } \\
\left(\mathrm{Mg} / \mathrm{m}^{3}\right)\end{array}$ & $\begin{array}{l}\text { Generalised } \\
\text { soil type }\end{array}$ & $\begin{array}{l}\text { Soil Classifications } \\
\text { [USCS \& BS 5390] }\end{array}$ \\
\hline $1(\mathrm{PS})$ & $\begin{array}{l}2.370 \\
\text { to } 2.777\end{array}$ & $\begin{array}{l}\text { Sand with silty } \\
\text { particles }\end{array}$ & Silty sand (SM) \\
\hline 2 (GS) & $\begin{array}{l}2.380 \\
\text { to } 2.483\end{array}$ & $\begin{array}{c}\text { Gravelly soil } \\
\text { with clay mineral } \\
\text { compositions }\end{array}$ & $\begin{array}{l}\text { Clayey sand with gravel } \\
\text { (SC) }\end{array}$ \\
\hline $3(\mathrm{WL})$ & $\begin{array}{l}2.453 \\
\text { to } 2.739\end{array}$ & $\begin{array}{l}\text { Clay with gravel } \\
\text { particles }\end{array}$ & $\begin{array}{l}\text { Gravelly clays of } \\
\text { intermediate plasticity } \\
\text { (CI) }\end{array}$ \\
\hline $4(\mathrm{PL})$ & $\begin{array}{l}2.640 \\
\text { to } 2.73\end{array}$ & $\begin{array}{l}\text { Sand with clay } \\
\text { composition }\end{array}$ & Clayey sand (SC) \\
\hline
\end{tabular}

Note:

$1=\mathrm{AP} 108 / 15 ; 2=\mathrm{AP} 108 / 20 ; 3=\mathrm{KL} 30 ; 4=\mathrm{AP} 104 / 5$; PS = Poor Soil $;$ GS = Good Soil; WL = Waterlogged; PL = Pile

- $\mathrm{SC}$ and SM = Unified Soil Classification System (USCS)

- $\mathrm{CI}$ = BS 5930 classification system

Table 11: Specific gravities of some soils

\begin{tabular}{ccc}
\hline S/No & Type of Soil & $\begin{array}{c}\text { Specific gravity } \\
(\mathrm{Gs}) \text { range }\left(\mathrm{Mg} / \mathrm{m}^{3}\right)\end{array}$ \\
\hline 1 & Gravel & $2.65-2.68$ \\
2 & Quartz Sands $^{\prime}$ & $2.64-2.66$ \\
3 & Silty & $2.67-2.73$ \\
4 & Clay & $2.70-2.90$ \\
5 & Chalk & $2.60-2.75$ \\
6 & Loess & $2.65-2.73$ \\
7 & Peat / Organic soils & $1.30-1.90$ \\
& Clay soil mineral compositions 2.0$)$ \\
\hline 8 & Clay $^{-}$K-Feldspars & \\
9 & Montmorillonite $^{(2)}$ & $2.54-2.57$ \\
10 & Illite $^{(2)}$ & $2.35-2.70$ \\
11 & Kaolinite $^{(2)}$ & $2.6-3.0$ \\
12 & Biotite $^{(1)}$ & $2.6-2.68$ \\
\hline
\end{tabular}

\section{References:}

(1) Lambe and Whitman, 1969; (2) Mitchell, 1993

Source: Das (2016); Das and Sobhan (2018)

\subsection{The Direct Shear Testing}

The direct shear test showed that the friction angle $(\phi)$ value was greater than that of cohesion $(c)($ i.e $\phi>\emptyset)$ at $5.2 \mathrm{~m}$ depth, and friction angle $(\phi)$ was less than cohesion $(c)$ (i.e $\phi<\emptyset$ ) at $10.4 \mathrm{~m}$ depth, despite both soil strata being classified as clayey-sand soils. This showed an increased clay and silt composition in the bottom soil strata since they induce the sand with increased interlocking behaviour/cohesion $[63,68]$. The obtained results of cohesion (c) and angle of internal friction $(\phi)$ were used in computing the soil's bearing capacities, pile skin and end-bearing resistances using the lateral earth pressure coefficient. Equations (17) to (19) are bearing capacity formulae for Terzaghi, and Meyerhof's vertical and inclined loads respectively whereas Eq. (20) and (21), and Eq. (22) and (23) are for predominantly clay and sandy soils respectively.

$\mathrm{q}_{\mathrm{u}}=\mathrm{cN}_{\mathrm{c}} \mathrm{S}_{\mathrm{c}}+0.5 \gamma_{\mathrm{t}} \mathrm{BN}_{\gamma} \mathrm{S}_{\gamma}+\gamma_{\mathrm{t}} \mathrm{D}_{\mathrm{f}} \mathrm{N}_{\mathrm{q}}$

$\mathrm{q}_{\mathrm{u}}=\mathrm{cN}_{\mathrm{c}} \mathrm{S}_{\mathrm{c}} \mathrm{d}_{\mathrm{c}}+\mathrm{q}_{\mathrm{o}} \mathrm{N}_{\mathrm{q}} \mathrm{S}_{\mathrm{q}} \mathrm{d}_{\mathrm{q}}+0.5 \gamma \mathrm{BN}_{\gamma} \mathrm{S}_{\gamma} \mathrm{d}_{\gamma}$

$\mathrm{q}_{\mathrm{u}}=\mathrm{cN}_{\mathrm{c}} \mathrm{d}_{\mathrm{c}} \mathrm{i}_{\mathrm{c}}+\mathrm{q}_{\mathrm{o}} \mathrm{N}_{\mathrm{q}} \mathrm{d}_{\mathrm{q}} \mathrm{i}_{\mathrm{q}}+0.5 \gamma B \mathrm{BN}_{\gamma} \mathrm{d}_{\gamma} \mathrm{i}_{\gamma}$

$$
\begin{aligned}
& \mathrm{f}_{\mathrm{s}}=\alpha \mathrm{S}_{\mathrm{u}}=\alpha\left(\frac{\mathrm{qu}_{\mathrm{u}}}{2}\right)=\alpha\left(\frac{12 \times \mathrm{N}_{55}}{2}\right) \\
& \mathrm{q}_{\mathrm{b}}=N_{c} \mathrm{~S}_{\mathrm{u}} \omega=\left[N_{c}\left(\frac{12 \times \mathrm{N}_{55}}{2}\right) \omega\right] \\
& f_{s}=K \bar{q} \tan \delta=\left[1-\left(\bar{q} \sin \emptyset^{\prime} x \tan \emptyset^{\prime}\right)\right] \\
& q_{b}=N_{q} \bar{q}
\end{aligned}
$$

Where:

$\mathrm{f}_{\mathrm{s}}=$ shaft skin resistance; and $\alpha=$ Adhesion factor

$S_{u}=$ average undrained shear strength

$q_{u}=$ unconfined compressive strength

$q_{b}=$ end bearing resistance; and $\delta=$ friction angle

$N_{c}, \mathrm{~N}_{\mathrm{q}} \& \mathrm{~N}_{\gamma}=$ Terzaghi's bearing capacity factors

$\bar{q}=$ effective overburden pressures

\begin{tabular}{|c|c|c|c|c|c|c|}
\hline $\begin{array}{l}\text { Depth } \\
\text { (m) }\end{array}$ & $\begin{array}{c}\text { Width } \\
\text { (m) }\end{array}$ & $\begin{array}{c}\text { Clay and } \\
\text { silt }(\%) \\
\text { from PSD* }\end{array}$ & $\begin{array}{c}\text { Bulk } \\
\text { Density } \\
\left(\mathrm{Mg} / \mathrm{m}^{3}\right)\end{array}$ & $\begin{array}{c}\mathrm{c} \\
(\mathrm{kPa})\end{array}$ & $\begin{array}{l}\phi \\
\left({ }^{\circ}\right)\end{array}$ & $\begin{array}{c}\boldsymbol{q}_{\text {all }} \\
(\mathrm{kPa})\end{array}$ \\
\hline 5.20 & 1 & $43.8 \%$ & 1.790 & 12.3 & 21 & 342 \\
\hline 10.40 & 1 & $63.2 \%$ & 1.745 & 18.5 & 15 & 348 \\
\hline
\end{tabular}

$\emptyset^{\prime}=$ effective internal friction angle from SPT test

$K=$ lateral earth pressure coefficient

Table 12: Insitu direct shear results for AP 104/5

\subsection{The One-Dimensional Consolidation Testing}

The consolidation $C_{v}$ value of $0.0042 \mathrm{~cm}^{2} / \mathrm{s}$ in the range of 0.00032 to $0.0032 \mathrm{~cm}^{2} / \mathrm{s}$ corresponded to a medium consolidation category, typical of $15-25 \%$ clays of the low plastic clay (CL) as per USCS system; whereas, the $m_{v}$ values of $0.187 \mathrm{~m}^{2} / \mathrm{MN}$ in the range of $0.25-0.125$ (Table 15) and 0.1-0.3 (Table 16), corresponded to stiff or firm clays of consolidated lake deposits or lacustrine/swampy soils of medium compressibility properties of 0.05 to 0.15 compression index $\left(C_{c}\right)[63,69]$.

Table 13: Insitu consolidation results for AP 104/5

\begin{tabular}{cccccc}
\hline $\begin{array}{c}\text { Test Depth } \\
(\mathrm{m})\end{array}$ & $\begin{array}{c}\mathrm{e}_{\mathrm{o}} \\
(-)\end{array}$ & $\begin{array}{c}\gamma_{\mathrm{b}} \\
\left(\mathrm{Mg} / \mathrm{m}^{3}\right)\end{array}$ & $\begin{array}{c}\mathrm{c}_{\mathrm{v}} \\
\left(\mathrm{cm}^{2} / \mathrm{s}\right)\end{array}$ & $\begin{array}{c}\mathrm{m}_{\mathrm{v}} \\
\left(\mathrm{m}^{2} / \mathrm{MN}\right)\end{array}$ & $\begin{array}{c}\mathrm{p}_{\mathrm{o}} \\
(\mathrm{kPa})\end{array}$ \\
\hline $10.4-10.7$ & 0.752 & 1.745 & 0.0042 & 0.187 & 201
\end{tabular}

Where:

$\mathrm{e}_{\mathrm{o}}=$ Initial void ratio; $\gamma_{\mathrm{b}}=$ Initial bulk density;

$\mathrm{c}_{\mathrm{v}}=$ Coefficient of consolidation

$\mathrm{m}_{\mathrm{v}}=$ Coefficient of volume compressibility;

$\mathrm{p}_{\mathrm{o}}=$ Pre-consolidation pressure

Table 14: Coefficient of consolidation

\begin{tabular}{cccc}
\hline $\begin{array}{c}\boldsymbol{C}_{\boldsymbol{v}} \text { Range } \\
\mathrm{cm}^{2} / \mathrm{s}\end{array}$ & Category & $\begin{array}{c}\text { Typical } \\
\text { material }\end{array}$ & $\begin{array}{c}\text { Soil classification } \\
\text { (USCS) }\end{array}$ \\
\hline$<0.000032$ & Very Low & - & - \\
\hline $\begin{array}{c}0.000032 \\
\text { to } 0.00032\end{array}$ & Low & $>25 \%$ Clay & $\begin{array}{c}\text { Medium plasticity } \\
\text { clays (CL-CH), and } \\
\text { volcanic silt (MH) }\end{array}$ \\
\hline $\begin{array}{c}0.00032 \\
\text { to } 0.0032\end{array}$ & Medium & $\begin{array}{c}15-25 \% \\
\text { Clay }\end{array}$ & $\begin{array}{c}\text { Low plasticity } \\
\text { clay/mud (CL) }\end{array}$ \\
\hline $\begin{array}{c}0.0032 \\
\text { to } 0.032\end{array}$ & High & $<15 \%$ Silt & Organic silt (OL) \\
\hline$>0.032$ & Very High & - & - \\
\hline
\end{tabular}




\begin{tabular}{|c|c|c|c|}
\hline $\begin{array}{c}C_{v} \text { Range } \\
\mathrm{cm}^{2} / \mathrm{s}\end{array}$ & Category & $\begin{array}{l}\text { Typical } \\
\text { material }\end{array}$ & $\begin{array}{c}\text { Soil classification } \\
\text { (USCS) }\end{array}$ \\
\hline
\end{tabular}

Note:

$1 \mathrm{~m}^{2} /$ year $=5 / 15768 \mathrm{~cm}^{2} / \mathrm{s} ; \boldsymbol{C}_{v}=$ coefficient of consolidation

Source: George et al. (2006), and Carter and Bentley (2016) [Adapted from Holtz and Kovacs (1981)]

Table 15: Coefficient of volume compressibility

\begin{tabular}{cc}
\hline $\boldsymbol{m}_{\boldsymbol{v}}\left(\mathrm{m}^{2} / \mathrm{MN}\right)$ & Soil type \\
\hline $10.0-2.0$ & Peat \\
$2.0-0.25$ & Plastic clay \\
$0.25-0.125$ & (normally consolidated alluvial clays) \\
\hline $0.125-0.0625$ & Hard clay (boulder clays) \\
\hline
\end{tabular}

Note: $\boldsymbol{m}_{\boldsymbol{v}}=$ Coefficient of volume compressibility

Source: Smith (2014)

Table 16: Compression index and descriptions

\begin{tabular}{|c|c|c|c|}
\hline $\begin{array}{r}\boldsymbol{M}_{\boldsymbol{v}} \\
\mathrm{m}^{2} / \mathrm{MN}\end{array}$ & $C_{c}$ & $\begin{array}{l}\text { Category of } \\
\text { compression }\end{array}$ & Soil Material Indicated \\
\hline$<0.05$ & 0.025 & $\begin{array}{l}\text { Very Low } \\
\text { compression }\end{array}$ & $\begin{array}{l}\text { Hard over-consolidated } \\
\text { glacial till, hard clay \& } \\
\text { stiff weathered rocks. }\end{array}$ \\
\hline $\begin{array}{l}0.05 \\
\text { to } 0.1\end{array}$ & $\begin{array}{l}0.025 \\
\text { to } 0.05\end{array}$ & $\begin{array}{l}\text { Low } \\
\text { compression }\end{array}$ & $\begin{array}{l}\text { Stiff glacial till/boulder } \\
\text { clays, marls, very stiff } \\
\text { tropical residual clays. }\end{array}$ \\
\hline $\begin{array}{l}0.1 \\
\text { to } 0.3\end{array}$ & $\begin{array}{l}0.05 \\
\text { to } 0.15\end{array}$ & $\begin{array}{l}\text { Medium } \\
\text { compression }\end{array}$ & $\begin{array}{l}\text { Firm clays of } \\
\text { consolidated swampy or } \\
\text { lake/ lacustrine deposits, } \\
\text { glacial outwash clays, } \\
\text { weathered marls, firm } \\
\text { glacial till, normally } \\
\text { consolidated clays at } \\
\text { depth, firm tropical } \\
\text { residual clays. }\end{array}$ \\
\hline $\begin{array}{l}0.3 \\
\text { to } 1.5\end{array}$ & $\begin{array}{l}0.15 \\
\text { to } 0.75\end{array}$ & $\begin{array}{c}\text { High } \\
\text { compression }\end{array}$ & $\begin{array}{l}\text { Poorly consolidated } \\
\text { alluvial clays, estuarine } \\
\text { deposits \& sensitive clay }\end{array}$ \\
\hline$>1.5$ & $\begin{array}{r}0.75 \\
\text { to } 5+ \\
\end{array}$ & $\begin{array}{l}\text { Very High } \\
\text { compression }\end{array}$ & $\begin{array}{l}\text { Highly organic alluvial } \\
\text { clays, and peats. }\end{array}$ \\
\hline
\end{tabular}

Where:

$\boldsymbol{C}_{\boldsymbol{c}}=$ compression index; $\boldsymbol{m}_{\boldsymbol{v}}=$ coeff. of vol. compressibility;

$\boldsymbol{a}_{v}=$ coefficient of compressibility

$\boldsymbol{m}_{v}=\left(\frac{a_{v}}{1+e_{o}}\right)=\left[\left(\frac{\delta_{e}}{\delta_{p}}\right) x \frac{1000}{\left(1+e_{o}\right)}\right]$

Source: Carter \& Bentley (2016) [Adapted from Bowles (1997)]

\subsection{The Particle Size Distribution (Soil grading)}

The particle size analysis showed that the soils at the formation levels of the locations AP 108/15 were poorly graded silty sand (SM), AP 108/20 were well graded clayey sand with gravel (SC), AP 104/5 were poorly graded clayey sand (SC) using the USCS classification system; whereas, the soils at KL 30 were gap graded gravelly clay (CI) as per the BS 5930 classification system. The gradations and soil classifications confirmed the previously inconclusive descriptions from the Test trial pit and borehole soil strata, parts of the preliminary soil consistency descriptions by DPL, specific gravity soil generalisations, material type identifications using the consolidation's $m_{v}$ and $C_{v}$ values, and the general soil type descriptions under plasticity index (PI) interpretations as discussed in sections 3.1, 3.2, 3.6, 3.8 and 3.10 respectively $[58,61,63]$.
Table 17: Insitu soil grading summaries

\begin{tabular}{cccc}
\hline Site & Soil Grading & $\begin{array}{c}\text { Formation } \\
\text { Level }\end{array}$ & $\begin{array}{c}\text { Soil } \\
\text { Classification }\end{array}$ \\
\hline AP 108/15 & Poorly-graded & $2.75 \mathrm{~m}$ & SM (USCS) \\
\hline AP 108/20 & Well-graded & $3.50 \mathrm{~m}$ & SC (USCS) \\
\hline AP 104/5 & Poorly-graded & $\sim 12.80 \mathrm{~m}$ & SC (USCS) \\
\hline KL 30 & Gap-graded & $4.50 \mathrm{~m}$ & CI (BS 5930) \\
\hline
\end{tabular}

Note: USCS = Unified Soil Classification System;

BS $5930=$ British Soil Classification System

\subsection{The Plasticity Index Interpretations}

The Atterberg tests showed that the soils at location AP $108 / 15$ had PI values in range of $7-17$, corresponding to medium-plastic soils of cohesive silty-sand type, whereas AP 108/20, KL 30 and AP 104/5 had soils with PI values greater than 17 (>17), corresponding to high plastic soils of cohesive clay type. Meanwhile, all the above locations had Liquid Limit (LL) values less than $50(<50)$, corresponding to finegrained soils with low swell potentials. The PI and LL test value interpretations were used in complementing the final classification and grading descriptions of the fine-grained soils as discussed in section 3.9 above $[58,68]$.

Table 18: Insitu Atterberg limit summaries

\begin{tabular}{|c|c|c|c|c|c|}
\hline \multirow{2}{*}{ Site } & \multicolumn{2}{|c|}{ Atterberg Limits } & \multirow{2}{*}{$\begin{array}{l}\text { FFL } \\
(\mathrm{m})\end{array}$} & \multirow{2}{*}{\multicolumn{2}{|c|}{$\begin{array}{c}\text { PI value range } \\
\text { (From Table 19) }\end{array}$}} \\
\hline & LL PL & PI & & & \\
\hline (PS) & $24.7 \quad 12.5$ & 12.20 & 2.75 & & -17 \\
\hline$(\mathrm{GS})$ & $44.8 \quad 21.4$ & 23.4 & 3.50 & & \\
\hline (WL) & $38.4 \quad 16.6$ & 21.8 & 4.50 & & 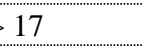 \\
\hline $4(\mathrm{PL})$ & $34.5 \quad 14.8$ & $19.7 \sim$ & $\sim 12.80$ & & \\
\hline \multicolumn{6}{|c|}{$\begin{array}{l}\text { Note: } \\
1=\text { AP } 108 / 15 ; 2=\text { AP } 108 / 20 ; 3=\text { KL } 30 ; 4=\text { AP } 104 / 5 ; \mathrm{PS}= \\
\text { Poor Soil; GS = Good Soil; WL = Waterlogged Location; PL = } \\
\text { Pile Location; FFL = Foundation's Formation Level } \\
\text { Table 19: PI interpretations and cohesiveness }\end{array}$} \\
\hline PI & Degree of $\mathrm{F}$ & Plasticity & $\begin{array}{r}\text { De } \\
\text { Cohe }\end{array}$ & $\begin{array}{l}\text { ree of } \\
\text { iveness }\end{array}$ & Soil Type \\
\hline 0 & Non-Pl & astic & Non- & ohesive & Sand \\
\hline$<7$ & Low Pl & astic & Partly & cohesive & Silt \\
\hline $7-17$ & Medium & Plastic & & hesive & Silty-Sand \\
\hline$>17$ & High Pl & lastic & & lesive & Clay \\
\hline
\end{tabular}

Source: Surendra and Sanjeev (2017)

Table 20: Atterberg Limits and Swell Potential

\begin{tabular}{ccc}
\hline $\begin{array}{c}\text { Liquid Limit } \\
(\text { LL })\end{array}$ & $\begin{array}{c}\text { Plasticity Index } \\
(\mathrm{PI})\end{array}$ & $\begin{array}{c}\text { Swell Potential } \\
(\mathrm{SP})\end{array}$ \\
\hline$<50$ & $<25$ & Low \\
\hline $50-60$ & $25-35$ & Marginal \\
\hline$>60$ & $>35$ & High \\
\hline
\end{tabular}

Source: Pitts (1984); Kalantari (1991)

\subsection{The Chemical Analysis Tests}

The chemical tests were done as a conclusive test following the preliminary soil resistivity test in section 3.4 above, to determine the insitu $\mathrm{pH}$, and presence of corrosioncausing sulphates and chlorides, in describing the insitu environmental exposure conditions as summarised in the Tables 21 and 22 below. 
The $\mathrm{SO}_{4}$ results showed that the soils at locations AP $108 / 15$, AP $108 / 20$ and AP 104/5 had values $\leq 3000 \mathrm{mg} / \mathrm{kg}$ ( $\leq 0.3 \%$ by weight) corresponding to XA1 exposure condition of slightly aggressive chemical environments; whereas, the KL 30 ground water had values of $\mathrm{SO}_{4}>600$ ppm, corresponding to XA2 exposure condition of moderately aggressive chemical environment as shown in Tables 21 and 22 below.

The $\mathrm{pH}$ values for AP 108/15 and AP 108/20 were 5.38 and 5.22 respectively, corresponding to XA2 exposure condition of moderately aggressive chemical environment, whereas KL 30 and AP 104/5 had pH values of 6.27 and 6.10 respectively, corresponding to XA1 exposure condition of slightly aggressive chemical environments. Thus, the chemical analysis and $\mathrm{pH}$ conditions were reconciled to provide XA2 exposure conditions of moderately aggressive chemical environment for all locations as per BS EN 206 (2013).

Sulphate Resistant Cements (SRC) of strength class $42.5 \mathrm{~N}$ and a $3.5 \%$ limited $\mathrm{C}_{3} \mathrm{~A}$ (chloro-aluminate) content were used under moderate water-cement ratios of 0.40 to 0.50 in order to inhibit the effects of chlorides forming insoluble chloro-aluminates $\left(\mathrm{C}_{3} \mathrm{~A}\right)$ upon combining with the Tricalcium Aluminate $\left(3 \mathrm{CaO} \cdot \mathrm{Al}_{2} \mathrm{O}_{3}\right)$ in concrete.

Table 21: Insitu chemical test results

\begin{tabular}{ccccc}
\hline Site & $\begin{array}{c}\boldsymbol{S O}_{4}{ }^{2-} \text { content } \\
(\% \text { by weight })\end{array}$ & $\begin{array}{c}\boldsymbol{C l}^{-} \text {content } \\
(\mathrm{g} / \mathrm{l})\end{array}$ & $\begin{array}{c}\mathrm{pH} \\
\text { value }\end{array}$ & $\begin{array}{c}\text { Sample } \\
\text { type }\end{array}$ \\
\hline 1 (PS) & $\begin{array}{c}0.05 \% \\
(500 \mathrm{ppm})\end{array}$ & $\begin{array}{c}0.007 \\
(7 \mathrm{ppm})\end{array}$ & 5.38 & Soil \\
\hline $2(\mathrm{GS})$ & $\begin{array}{c}0.06 \% \\
(600 \mathrm{ppm})\end{array}$ & $\begin{array}{c}0009 \\
(9 \mathrm{ppm})\end{array}$ & 5.22 & Soil \\
\hline $3(\mathrm{WL})$ & $\begin{array}{c}0.0686 \% \\
(686 \mathrm{ppm})\end{array}$ & $\begin{array}{c}10 \\
(10,000 \mathrm{ppm})\end{array}$ & 6.27 & $\begin{array}{c}\text { Ground } \\
\text { water }\end{array}$ \\
\hline $4(\mathrm{PL})$ & 0 & $\begin{array}{c}0.021 \\
(21 \mathrm{ppm})\end{array}$ & 6.10 & Soil \\
\hline
\end{tabular}

Where: $1 \mathrm{~g} / \mathrm{L}=1000 \mathrm{ppm}$; and $1 \mathrm{ppm}=1 \mathrm{mg} / \mathrm{L}=0.001 \mathrm{~g} / \mathrm{L}$

Table 22: Measured $\mathrm{SO}_{4}$ results interpretations

\begin{tabular}{lccc}
\hline Site & $\begin{array}{c}\text { Measured } \\
\mathrm{SO}_{4}(\mathrm{ppm})\end{array}$ & $\begin{array}{c}\text { Limiting values of } \\
\mathrm{SO}_{4}(\mathrm{ppm}) *\end{array}$ & $\begin{array}{c}\text { Exposure } \\
\text { condition* }\end{array}$ \\
\hline 1 (PS) & 500 & $\geq 2000$ and $\leq 3000$ (soil) & XA1 \\
\hline 2 (GS) & 600 & $\geq 2000$ and $\leq 3000$ (soil) & XA1 \\
\hline $3(\mathrm{WL})$ & 686 & $>600$ and $\leq 3000$ (water) & XA2 \\
\hline $4(\mathrm{PL})$ & 0 & $\geq 2000$ and $\leq 3000$ (soil) & XA1 \\
\hline NB: 1 = AP 108/15; 2 = AP $108 / 20 ; 3=$ KL $30 ; 4=$ AP 104/5; \\
PS = Poor Soil; GS = Good Soil; WL = Waterlogged Location; \\
PL = Pile Location \\
* Source: BS EN 203 (2013)
\end{tabular}

\subsection{Concrete cube compressive strength Tests}

The concrete cube compressive strength test was done as per BS EN 12390-1 (2012) and BS EN 12390-2 (2009), to determine the 7-day and 28-day strengths as a confirmatory quality control test of the $25 \mathrm{MPa}$ design strength using $42.5 \mathrm{~N}$ Sulphate Resistant Cement as shown in Table 23 below. The results showed that all the locations had 7-day test strength values within the $104.2-123.36 \%$ range of the 25 $\mathrm{MPa}$ design value, and 28-day test strength values in the range of $155.12-211.08 \%$ of the $25 \mathrm{MPa}$ design value. The compressive test values were used to confirm and provide assurance to the foundation's design concrete strength value of $25 \mathrm{MPa}$ using $42.5 \mathrm{~N} \mathrm{SRC}$ as a remedy to the sulphate and chloride attacks, as discussed in section 3.11.

Table 23: Insitu compressive concrete cube results

\begin{tabular}{|c|c|c|c|c|}
\hline \multirow{3}{*}{ Site } & \multicolumn{4}{|c|}{\begin{tabular}{|c} 
Tested Cube strength values \\
[For a 28-day Design Strength (DS) of $25 \mathrm{MPa}]$
\end{tabular}} \\
\hline & \multicolumn{2}{|c|}{ 7-day strength } & \multicolumn{2}{|c|}{ 28-day strength } \\
\hline & $\begin{array}{l}\text { Results } \\
(\mathrm{MPa})\end{array}$ & $\%$ of DS & $\begin{array}{c}\text { Results } \\
(\mathrm{MPa})\end{array}$ & $\%$ of DS \\
\hline 1 (PS) & 29.81 & 119 & 43.31 & $173.24 \%$ \\
\hline $2(\mathrm{GS})$ & 26.05 & $104.20 \%$ & 38.78 & $155.12 \%$ \\
\hline $3(\mathrm{WL})$ & 27.71 & $110.84 \%$ & 40.08 & $160.32 \%$ \\
\hline $4(\mathrm{PL})$ & 30.84 & $123.36 \%$ & 52.77 & $211.08 \%$ \\
\hline
\end{tabular}

\subsection{Static Load Tests}

The insitu static load tests were done to determine the insitu displacement and load capacity values of the foundations under tension/uplift, compression, and lateral load tests in conformity to IEC 61773 [57] as shown in Tables 24 to 26.

The static load test results showed that the location AP 104/5 exhibited maximum displacement values of $0.09 \mathrm{~mm},-0.83$ $\mathrm{mm}$ and $2.39 \mathrm{~mm}$ under tension, compression and lateral loading respectively; while locations AP 108/15, AP 108/20 and KL 30 exhibited maximum tension displacement values of $0.83 \mathrm{~mm}, 0.19 \mathrm{~mm}$ and $4.74 \mathrm{~mm}$ respectively. The limiting reference displacement values were $25 \mathrm{~mm}$ for both tension and compression loading tests, and $50 \mathrm{~mm}$ for the lateral loading test. The static load results were used in determining the slope $\left(C_{1}\right)$ of the hyperbolic model graph's empirical line equation using Eq. (24) and (25) below, and in the calculation of the insitu foundation's load capacity $\left(R_{c}\right)$ using ChinKondner extrapolation (1971) as per Eq. (26) below.

$$
\begin{aligned}
& y=m x+c \quad \text { (Line of best fit) } \\
& \text { Slope } \Rightarrow C_{1}=\frac{d}{d x}(y=m x+c) \\
& \text { Insitu Load Capacity } \Rightarrow \mathrm{R}_{\mathrm{c}}=\frac{1}{\mathrm{C}_{1}}(\text { in } \mathrm{kN})
\end{aligned}
$$

The actual insitu load capacities of the test-foundations under static load methods were from $105.29 \%$ to $249.14 \%$ fraction of the prescriptive design values, which reaffirmed the conclusion that the load capacity results of the insitu-tested full-scale foundations exceeded the prescriptively designed load capacity values, as shown in Table 26. 


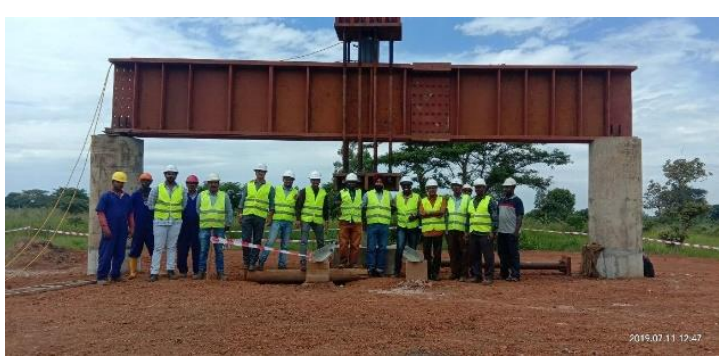

Fig.1: Insitu static axial tension/uplift load test

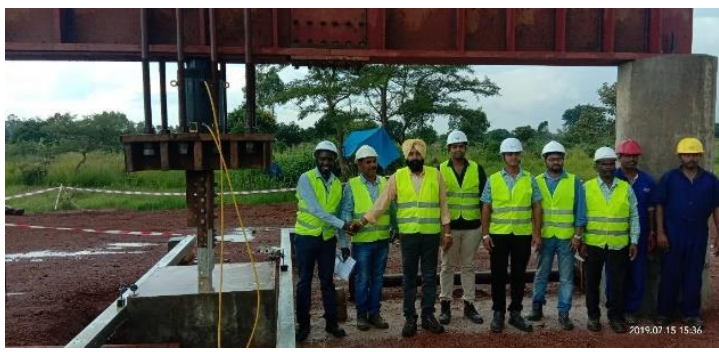

Fig.2: Insitu static axial compression load test

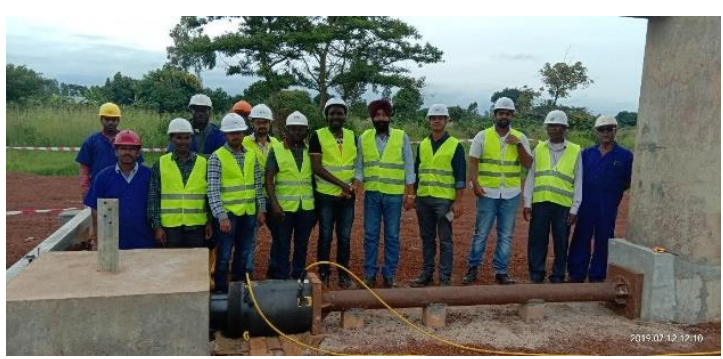

Fig.3: Insitu static lateral load test

Table 24: Insitu static load test summaries

\begin{tabular}{c|ccc|cc|c|c}
\hline \multirow{2}{*}{$\begin{array}{c}\text { Site } \\
\text { Location }\end{array}$} & \multicolumn{2}{|c|}{ Insitu Maximum } & \multicolumn{3}{|c}{ IEC 61773 (1996) } \\
& T & T & C & L & T & C & L \\
\hline AP 108/15 & 0.83 & - & - & & & \\
AP 108/20 & 0.19 & - & - & 25 & 25 & 50 \\
KL 30 & 4.74 & - & - & & & \\
AP 104/5 & 0.09 & -0.83 & 2.39 & & & \\
\hline
\end{tabular}

Where:

$\mathrm{T}=$ Static Axial Tension/Uplift Loading Test

$\mathrm{C}=$ Static Axial Compression Loading Test

$\mathrm{L}=$ Static Lateral Loading Test

Table 25: Slope readings for insitu static load tests

\begin{tabular}{cccc}
\hline \multirow{2}{*}{$\begin{array}{c}\text { Site } \\
\text { Location }\end{array}$} & $\begin{array}{c}\text { Tension } \\
\text { Test }\end{array}$ & $\begin{array}{c}\text { Compression } \\
\text { Test }\end{array}$ & $\begin{array}{c}\text { Lateral } \\
\text { Test }\end{array}$ \\
\hline AP 108/15 & 0.9996 & - & - \\
AP 108/20 & 1.3568 & - & - \\
KL 30 & 0.8755 & - & - \\
AP 104/5 & 0.7223 & 0.5019 & 5.2591 \\
\hline
\end{tabular}

Table 26: Insitu foundation load capacities

\begin{tabular}{|c|c|c|c|c|c|c|}
\hline \multirow{3}{*}{ Site } & \multicolumn{6}{|c|}{ Load Capacities } \\
\hline & \multicolumn{2}{|c|}{$\begin{array}{l}\text { Tension } \\
\text { Test }(\mathrm{kN})\end{array}$} & \multicolumn{2}{|c|}{$\begin{array}{c}\text { Compression } \\
\text { Test }(\mathrm{kN})\end{array}$} & \multicolumn{2}{|c|}{$\begin{array}{c}\text { Lateral } \\
\text { Test }(\mathrm{kN})\end{array}$} \\
\hline & $\begin{array}{l}\text { Insitu } \\
\text { Load }\end{array}$ & UDL & $\begin{array}{l}\text { Insitu } \\
\text { Load }\end{array}$ & UDL & $\begin{array}{l}\text { Insitu } \\
\text { Load }\end{array}$ & UDL \\
\hline $1(\mathrm{PS})$ & 1000.4 & 945.36 & - & - & - & - \\
\hline $2(\mathrm{GS})$ & 737.03 & 594.45 & - & - & - & - \\
\hline $3(\mathrm{WL})$ & 1142.2 & 962.30 & - & - & - & - \\
\hline
\end{tabular}

\begin{tabular}{l|l|l|l|l|l|l|}
$4(\mathrm{PL})$ & 1384.5 & 555.69 & 1992.43 & 1077.1 & 190.15 & 180.6 \\
\hline
\end{tabular}

Note: 1 = AP 108/15; 2 = AP 108/20; 3 = KL 30; 4 = AP

104/5; PS = Poor Soil; GS = Good Soil; WL = Waterlogged

Location; PL = Pile Location; UDL = Ultimate Design Load

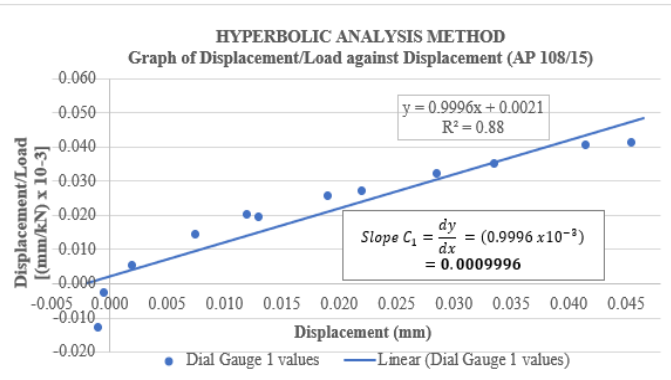

Fig.4: AP 108/15- Hyperbolic graph in uplift

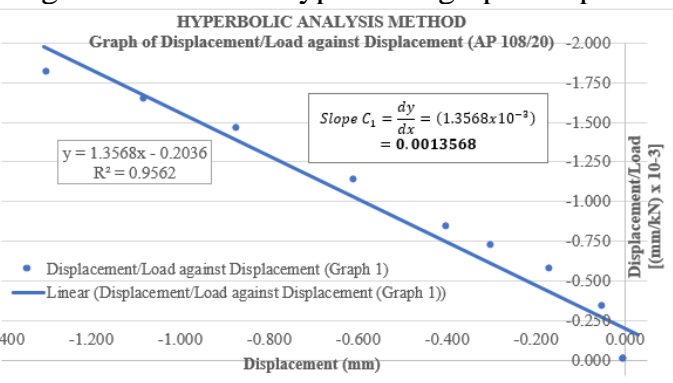

Fig.5: AP 108/20- Hyperbolic graph for uplift

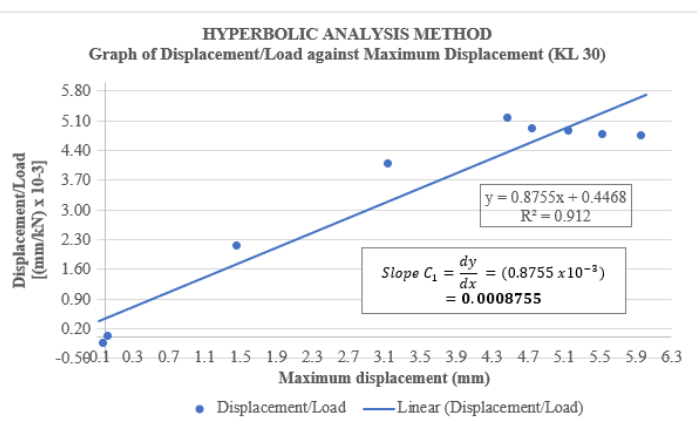

Fig.6: KL 30- Hyperbolic graph for uplift

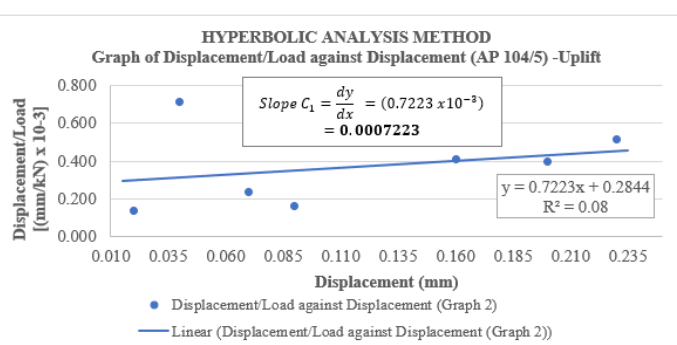

Fig.7: AP 104/5- Hyperbolic graph for uplift 


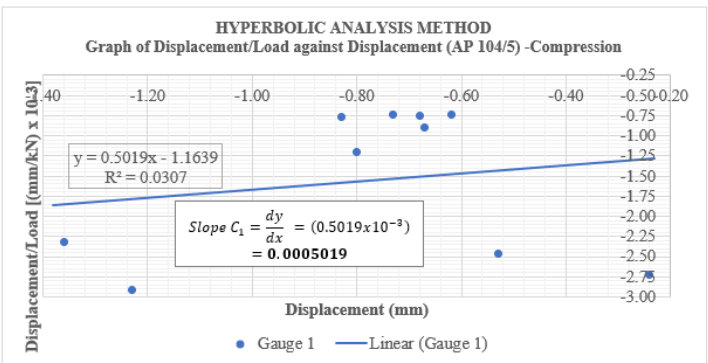

Fig.8: AP 104/5- Hyperbolic graph for compression

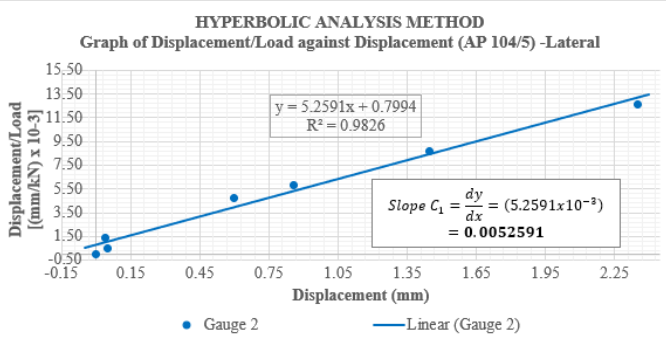

Fig.9: AP 104/5- Hyperbolic graph for lateral

\section{CONCLUSION}

Based on the reviews of the experimental results, the following conclusions were reached:

The insitu load capacities of the test foundations under static load were $105.29 \%$ to $249.14 \%$ fraction of the theoretical load capacities, confirming that the prescriptive design approaches use equations and methods governing a linear-elastic boundary in the design value extrapolations instead of the more-realistic plastic and non-linear approach.

The maximum insitu displacement values from static load tests differed significantly from that of the prescriptive design and technical specifications by being $0.36 \%$ to $18.96 \%$ fraction of the $25 \mathrm{~mm}$ prescriptive limit under uplift and $3.32 \%$ fraction of the prescriptive $25 \mathrm{~mm}$ under compression and $4.78 \%$ fraction of the prescriptive $50 \mathrm{~mm}$ limit under lateral test. These displacements were less than $19 \%$ of the prescriptive values as verified by the insitu static load tests of the foundations.

Due to the acidic soils and ground water, the use of $42.5 \mathrm{~N}$ Sulphate Resistant Cement (SRC) led to very high compressive strength provided for the concrete foundations due to lack of a low grade $32.5 \mathrm{~N} \mathrm{SRC}$ in Uganda. This led to an overdesign in the concrete's compressive strength ranging from $104.2 \%$ to $123.36 \%$ of the design strength at 7 day and $155.12 \%$ to $211.08 \%$ at 28 days.

\section{ACKNOWLEDGMENTS}

The author would like to thank Sinohydro Corporation Ltd, Kalpataru Power Transmission Ltd, and GOPAInternational Energy Consultants for their contributions towards the research at the Karuma Interconnection Project in Uganda.

\section{REFERENCES}

[1] Manjriker Gunaratne (2014) Foundation Engineering Handbook, $2^{\text {nd }}$ Edition. ISBN-13: 978-14398-92787. CRC Press, Taylor \& Francis, Florida, USA, pp 105 - 379

[2] Kaushik C.P., Bhavikatti S.S. \& Anubha Kaushik (2010) Basic Civil and Environmental Engineering. ISBN 13: 978-81-224-2850-6. New Age International (P) Ltd., New Delhi, India, pp 22 - 31.

[3] Sivakugan N. and Das, Braja M. (2011) Geotechnical Engineering- A Practical Problem-Solving Approach, ISBN-13: 978-1-60427-016-7. J. Ross Publishing, Inc., Florida, USA, pp 289 - 483.

[4] Federal Highway Administration (1992) Static Testing of Deep Foundations, Publication No. FHWA-SA-91-042. (Kyfor Z. G., Schnore A. R., Carlo T. A., and Baily P. F., eds), Soil Mechanics Bureau, NY State Department of Transportation, New York, USA, pp 1 - 165.

[5] Byrne, G. and Berry, A. (2008) A Guide to Practical Geotechnical Engineering in Southern Africa, $4^{\text {th }}$ Edition. Franki Africa (Pty) Ltd Cape Town, South Africa, pp 156 - 166.

[6] Monnet, Jacques (2015) In Situ Tests in Geotechnical Engineering. ISBN: 978-1-848-21849-9. ISTE Ltd, London, UK, pp 73 - 104.

[7] Cockerill, P., Nutter, J., Bradshaw, K., Ehrman, T., and Witney, J. (2017). East African Piling Limited Company Profile, March 2017, Available at: www.eastafricanpiling.com, Kampala, Uganda, pp 1 - 10.

[8] Tomlinson, M. J. and Boorman, R. (2001) Foundation Design and Construction, 7th Edn, ISBN: 0130-31180-4. Pearson Education, Essex CM20 2JE, England, pp. 1 - 547.

[9] Mosley Bill, John Bungey and Ray Hulse (2007) Reinforced Concrete Design to Eurocode 2, $6^{\text {th }}$ Edition. ISBN-13: 9780230500716 , Palgrave Macmillan Press, NY, USA, pp 15 - 308.

[10]Bayliss C.R. and Hardy B.J. (2011) Transmission and Distribution Electrical Engineering, $4^{\text {th }}$ Edn, ISBN 978-0-08-096912-1. Elsevier Press, Oxford, UK, pp 615 - 629.

[11]Emuriat, Julius Emmanuel (2017) Parametric study on analysis and design of secant pile for earthquake loading, ISBN: 978-620-2-30082-7. Scholar's Press, Mauritius, pp 1 - 90.

[12]Przewłócki, J., Dardzińska, I. and Świniański, J. (2005) Review of historical buildings' foundations, Géotechnique, 55 (5), pp. 363 - 372. doi: 10.1680/geot.55.5.363.66017

[13] Salgado, Rodrigo (2006) The engineering of foundations, $1^{\text {st }}$ Ed. ISBN13: 978-0072500585, McGraw-Hill, PA, USA, pp 21-896.

[14] An-Bin Huang and Hai-Sui Yu (2018) Foundation engineering Analysis and Design, 1st Edition, ISBN 13: 978-1-138-72079-4. CRS Press, Florida, USA, pp 189 - 239

[15]Lacasse, S. and Nadim, F. (1994). Reliability issues and future challenges in geotechnical engineering for offshore structures. Proc., 7th Int. Conf. on Behaviour of Offshore Structures, Cambridge, Massachusetts, pp. 9 - 38

[16] Gilbert, R. B. and Tang, W. H. (1995). "Model uncertainty in offshore geotechnical reliability." Proc. 27th Offshore Technology Conference, Houston, Texas, 557 - 567.

[17]Phoon, K. K and Kulhawy, F. H. (1999). Characterization of geotechnical variability. Canadian Geotechnical Journal, 36(4), 612 624.

[18] Whitman, R. V. (2000). Organizing and evaluating uncertainty in geotechnical engineering. Journal of Geotechnical and Geoenvironmental Eng., 126 (7), 583 - 593.

[19]Juang, C. H., Yang, S. H., Yuan, H., and Khor, E. H. (2004). Characterization of the uncertainty of the Robertson and Wride model for liquefaction potential evaluation. Soil Dynamics and Earthquake Engineering, 24(9), 771-780.

[20] Schuster, M.J., Juang, C. H., Roth, M.J.S., and Rosowsky, D. V. (2008). Reliability analysis of building serviceability problems caused by excavation. Géotechnique, 58(9), 743-749.

[21]Zhang, J., Zhang, L. M., and Tang, W. H. (2009). Bayesian framework for characterizing geotechnical model uncertainty. Journal of Geotechnical \& Geoenvironmental Engineering, 135 (7), 932 - 940

[22] Juang, C. H., Fang, S. Y., Tang, W. H., Khor, E. H., Kung, G. T., and Zhang, J. (2009). Evaluating model uncertainty of an SPT-based simplified method for reliability analysis for probability of liquefaction. Soils and Foundations, 49 (12), 135 - 152

[23]Zhang, J., Tang, W. H., Zhang, L. M., and Huang, H. W. (2012) Characterising geotechnical model uncertainty by hybrid Markov Chain Monte Carlo simulation. Computers and Geotechnics, 43, 26 - 36.

[24]Juang, C. H., Lei Wang, Sez Atamturktur, and Zhe Luo (2012). Reliability-based robust and optimal design of shallow foundations in 
cohesionless soil in the face of uncertainty. Journal of GeoEngineering, Vol. 7, No. 3, pp. 075-087, doi: 10.6310/jog.2012.7 (3).1.

[25]Wu, T., Tang, W. H., Sangrey, D. A., and Baecher, G. B. (1989). Reliability of offshore foundations - state-of-the-art. Journal of Geotechnical Eng., 115 (2), 157 - 178.

[26]Christian, J. T., Ladd, C. C., and Baecher, G. B. (1994). "Reliability applied to slope stability analysis." Journal of Geotechnical Engineering, 120 (12), 2180 - 2207.

[27]Phoon, K. K., Kulhawy, F. H., and Grigoriu, M. D. (2003a). Development of a reliability-based design framework for transmission line structure foundations. Journal of Geotechnical and Geoenvironmental Eng, 129 (9), 798 - 806

[28]Phoon, K. K., Kulhawy, F. H., and Grigoriu, M. D. (2003b). Multiple resistance factor design for shallow transmission line structure foundations. Journal of Geotechnical and Geoenvironmental Engineering, 129 (9), 807 - 818

[29]Fenton, G. A., Griffiths, D. V., and Williams, M. B. (2005). Reliability of traditional retaining wall design. Géotechnique, 55 (1), 55-62.

[30]Najjar, S. and Gilbert, R. (2009). Importance of lower-bound capacities in the design of deep foundations. Journal of Geotechnical \& Geoenvironmental Eng., 135 (7), 890 - 900.

[31]Wang, Y. (2011). Reliability-based design of spread foundations by Monte Carlo Simulations. Géotechnique, 61 (8), 677 - 685.

[32]Zhang, J., Zhang, L.M., and Tang, W.H. (2011). Reliability-based optimization of geotechnical systems. Journal of Geotechnical and Geoenvironmental Eng, 137 (12), 1211-1221.

[33] Venkatesh, K., Samadhiya, N. K. and Pandey, A. D. (2008). Approaches of analysis of ogee shaped barrage raft floor on varying foundation media. Proceedings of the $6^{\text {th }}$ International Conf. on case histories in geotechnical eng. and symposium, Arlington, VA, August 11-16, 2008.

[34]Rodrigo Salgado et al. (2008) Analysis, Design, Testing and Performance of Foundations, Aug $11^{\text {th }}-16^{\text {th }} 2008,6^{\text {th }}$ International Conf on Case Histories in Geotechnical Eng., pp. 1 - 23.

[35]Barvashov, V. A., Kharlamov, P. V., Naidenov, A. I. and Rytov, S. A. (2008). "Application of simplified models to qualitative geotechnical analysis" Proceedings of the $6^{\text {th }}$ Intern. Conf. on case histories in geotechnical eng. and symposium, Arlington, VA, August 11-16, 2008.

[36] Murad Abu-Farsakh, Khalid Alshibli, and Anand Puppala (2017) Advances in analysis and design of deep foundations. Proceedings of the $1^{\text {st }}$ GeoMEast International congress and exhibition, on sustainable civil infrastructures, ISBN 9783319616414, pp 1 - 300.

[37]Ministry of Energy and Mineral Development (2013) Technical Specifications for Karuma Hydropower Project and Associated Transmission Lines (MoE\&MD, 2013)- Part A: Technical Specifications for Transmission Works, Republic of Uganda, pp. 1 - 260

[38] Sriram, Kalaga and Prasad, Yenumula (2017) Design of electrical transmission line: structures and foundations, ISBN 9781315755687. CRC, London, UK, pp. 185-293.

[39]ASTM D2216-19 (2019) Standard test methods for laboratory determination of water (Moisture) content of soil and rock by mass, ASTM International, West Conshohocken, PA, 2019, www.astm.org

[40] ASTM D6913/D6913M-17 (2017) Standard test methods for particlesize distribution (Gradation) of soils using sieve analysis, ASTM International, West Conshohocken, PA, 2017, www.astm.org

[41] ASTM D2487-17 (2017) Standard practice for classification of soils for engineering purposes (Unified Soil Classification System), ASTM International, West Conshohocken, PA, 2017, www.astm.org

[42]ASTM D4318-17 (2017) Standard Test Methods for Liquid Limit, Plastic Limit, and Plasticity Index of Soils, ASTM International, West Conshohocken, PA, 2017, www.astm.org

[43] British Standards Institution (1990) BS 1377-2:1990. Methods of test for soils for civil engineering purposes. Classification tests. ISBN: 978 058017867 6, London, BSI

[44]British Standards Institution (1990) BS 1377-3:1990. Methods of test for soils for civil eng. purposes. Chemical and electro-chemical tests. ISBN $978058018370 X$, London, BSI

[45] ASTM G51-18 (2018) Standard test method for measuring pH of Soil for use in corrosion testing, ASTM International, West Conshohocken, PA, 2018, www.astm.org

[46] ASTM D4327-11 (2011) Standard test method for anions in water by suppressed ion chromatography, ASTM International, West
Conshohocken, PA, 2011, www.astm.org

[47]ASTM D7263-09 (2018) Standard test methods for laboratory determination of density (Unit Weight) of soil specimens, ASTM International, West Conshohocken, PA, 2018, www.astm.org

[48] ASTM D854-14 (2014) Standard test methods for specific gravity of soil solids by water pycnometer, ASTM International, West Conshohocken, PA, 2014, www.astm.org

[49]ASTM D3080/D3080M-11 (2011) Standard test method for direct shear test of soils under consolidated drained conditions, ASTM International, West Conshohocken, PA, 2011, www.astm.org

[50]British Standards Institution (1990) BS 1377-6:1990. Methods of test for soils for civil engineering purposes. Consolidation and permeability tests in hydraulic cells and with pore pressure measurement. ISBN 9780580185885 , London, BSI.

[51]ASTM D2435/D2435M-11 (2011) Standard test methods for onedimensional consolidation properties of soils using incremental loading, ASTM International, West Conshohocken, PA, 2011, www.astm.org

[52] Tomlinson, M. J. and Woodward, J. (2015) Pile design and construction practice, $6^{\text {th }}$ Edn, ISBN-13: 9781466592643. CRC Press, Taylor and Francis Group, Florida, USA, pp. 1 - 569.

[53] Hertlein, B. H., and Davis, A. G. (2006). Non-Destructive testing of Deep Foundations. ISBN 13: 9780470848500 , John Wiley \& Sons Ltd, West Sussex, England, pp 19 - 100.

[54] ASTM D1143/D1143M-07 (2013) e1, Standard test methods for deep foundations under static axial compressive load, ASTM International, West Conshohocken, PA, 2013, www.astm.org

[55]ASTM D3689/D3689M-07 (2013) e1, Standard test methods for deep foundations under static axial tensile load, ASTM International, West Conshohocken, PA, 2013, www.astm.org

[56]ASTM D3966/D3966M-07 (2013) e1, Standard test methods for deep foundations under lateral load, ASTM International, West Conshohocken, PA, 2013, www.astm.org

[57] IEC 61773 (1996) Overhead lines - Testing of foundations for structures, ASIN: B000XYS9YM, International Electrotechnical Commission, Geneva, Switzerland, www.iec.ch

[58]Das, Braja and Sobhan, Khaled (2018) Principles of Geotechnical Engineering. $9^{\text {th }}$ Edn. Cengage Learning, Boston, USA, pp $710-742$

[59] British Standards Institution (2005) BS EN ISO 22476-2:2005+A1:2011. Geotechnical investigation and testing. Field testing. Dynamic probing. ISBN: 9780580749131, London, BSI.

[60]Nilsson, T. (2012). Parameter approach from DPL Nilsson Test, $3^{\text {rd }}$ International Conf. on site characterisation, ISC-3, Taipei, Vol. 2, pp 1415-1418. Retrieved from www.nilsson.com.br

[61]Das, B. M. (2016) Principles of foundation engineering, $8^{\text {th }}$ Edn. ISBN13: 9781305081550 . Cengage, Boston, USA, pp 1 - 911.

[62] Roberge, Pierre (2000) Handbook of corrosion engineering, $1^{\text {st }}$ Edition, ISBN 9780070765160. McGraw-Hill, New York, USA, pp. 1 - 938.

[63] Smith, Ian (2014) Smith's element of soil mechanics, $9^{\text {th }}$ Edition, ISBN 9780470673393. John Wiley \& Son, West Sussex, UK, pp. 1- 459.

[64] Bowles, J. E. (1997). Foundation analysis and design, $5^{\text {th }}$ Edition, ISBN 13: 9780079122476. McGraw-Hill, Singapore, pp 1 - 343.

[65]Tuncer, Erdil Riza, and Lohnes, R.A. (1977) An engineering classification for basalt-derived lateritic soils., Eng. Geol., 4, 319 - 339.

[66]Roy, Surendra and Dass, Gurcharan (2014) Statistical models for the prediction of shear strength parameters at Sirsa, India. ISSN 0976-4399, doi: 10.6088/ijcser.201404040002, International Journal of Civil and Structural Engineering, 4 (4), pp 483 - 498.

[67]Roy, Surendra, and Sanjeev Kumar Bhalla, (2017) Role of Geotechnical Properties of Soil on civil engineering structures, resources and environment, Vol. 7, No. 4, e-ISSN: 2163-2634, pp. 103-109. doi 10.5923/j.re.20170704.0

[68]Das, B. M. (2019). Advanced soil mechanics. $5^{\text {th }}$ Edn. ISBN-13: 9780815379133. CRC Press, Taylor \& Francis, Florida, USA, pp 1 - 671.

[69]Carter, M., and Bentley, S. P. (2016) Soil properties and their correlation, 2nd Edition, ISBN: 978-1119130888. John Wiley and Sons Inc., West Sussex, UK, pg 1 - 217 .

Copyright (․ All rights reserved, including the making of copies unless permission is obtained from the copyright proprietors. 